\title{
Study on Early Enteral Nutrition after Gastrectomy and Its Nursing
}

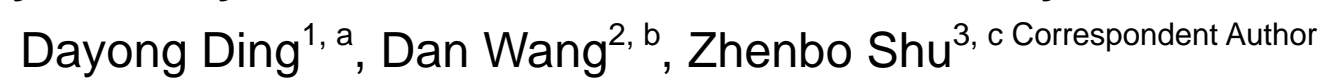

${ }^{1,3}$ Department of Gastrointestinal Colorectal and Anal Surgery, China-Japan Union Hospital, Jilin University, Changchun, 130033

${ }^{2}$ Operation Room of China-Japan Union Hospital, Jilin University, Changchun, 130033 aemail, bemail, ${ }^{\mathrm{c} e m a i l,}$

\section{Keywords: Early Enteral Nutrition, Gastrectomuy and Nursing}

\begin{abstract}
This paper summarized 42 cases of total gastrectomy for patients with early enteral nutrition care. Care focuses attention to psychological care, good care and nasal gastric tube and fixed bowel, strictly controlled input of nutrient solution, speed, temperature, diarrhea and bloating and other complications strengthen observation and care, good oral care. 42 patients were discharged, enteral nutrition complications.
\end{abstract}

\section{Introduction}

Patients with gastric cardia, gastric cancer, gastric cancer and other diseases for a long time due to illness consumption of water and electrolyte disorders and is often accompanied by malnutrition and other diseases, in addition to continuous decompression, after fasting, surgical trauma, aggravated easier nutritional deficiencies and loss of electrolytes. Of total gastrectomy in patients with early enteral nutrition support can reduce the incidence of complications in patients, improve the nutritional status of the patient, it is conducive to the rehabilitation of patients.

Total gastrectomy, long-term parenteral nutrition, lack of food in the intestine stimulation, can cause intestinal mucosal atrophy, increased bacterial translocation, intestinal barrier dysfunction, decreased immune function, prone to complications, especially anastomotic leakage, handle tricky. Early enteral nutrition is an effective way to maintain the intestinal barrier [2], a number of clinical studies have shown that, compared with delayed enteral nutrition, early enteral nutrition can significantly reduce mortality and infection rates, increased nutrient intake, reduce hospitalization cost. Total gastrectomy early body in a high catabolic state aggravated malnutrition, early postoperative nutritional support is one of the important therapeutic measures to prevent complications and promote the rehabilitation of patients. Injection early postoperative enteral nutrition, effective protection of intestinal mucosal barrier, maintain intestinal integrity, promoting recovery of bowel movement as soon as possible. Summarize our department 48 cases of total gastrectomy in patients with gastric nasal enteral nutritional support experience, are as follows.

\section{Nutrition Method}

Before the complex catheter tip inserted into the intestine Marche silicone nose ordinary silicone tube head a second side of the hole, making it integral with the tube, with the preoperative tube into the stomach, intestine and retained within the nose guide wire technique the nose then placed at a distance from the bottom of the jejunum intestinal anastomosis population of about $30 \sim 40 \mathrm{~cm}$. Note intubation action to slow, so as not to damage the intestinal mucosa, and the inspection process in place Have twisted or knotted. The first postoperative day Applied Nutrition enteral nutrition pumps.

Using intermittent feeding and continuous infusion. Since the first day after the nasal feeding tube test drops of warm saline $250 \mathrm{~mL}$ (with heater), the first two days after surgery by intestinal nutrient infusion pump from the nose \& Poor's 100 force $(1000 \mathrm{~mL}$ containing protein $40 \mathrm{~g}$, fat $17 \mathrm{~g}$ sugar class $176 \mathrm{~g}$. energy $4250 \mathrm{~kJ} / \mathrm{L}$, the concentration of osmotic $440 \mathrm{mmol} / \mathrm{L}$ ) $500 \mathrm{~mL}, 40 \mathrm{~mL} / \mathrm{h}$. 3 days after the full energy $(1000 \mathrm{~mL}$ containing protein $40 \mathrm{~g}$, fat 39g, 123g sugar and cellulose $75 \mathrm{~g}$. Energy $4184 \mathrm{~kJ} / \mathrm{L}$, osmotic concentration of $250 \mathrm{mmol} / \mathrm{L}$ ) $1000 \mathrm{~mL}$, speed $80 \sim 120 \mathrm{~mL} / \mathrm{h}$, 4 to 7 days 
infusion $1500 \mathrm{~mL}(80 \sim 120 \mathrm{~mL} / \mathrm{h})$, with a shortage of liquid from the peripheral vein of energy supply, ensure energy intake $105 \sim 126 \mathrm{~kJ}(/ \mathrm{kg} \bullet \mathrm{d})$. Nutrient solution temperature was controlled at $37 \sim 38{ }^{\circ} \mathrm{C}$.

The method of parenteral nutrition for 1-6 days after the first day infusion of fat emulsion, compound amino acid and glucose, the heat of $125 \mathrm{~kJ}(/ \mathrm{kg} \bullet \mathrm{d})$, nitrogen is $0.2 \mathrm{~g}(/ \mathrm{kg} \bullet \mathrm{d})$ and a variety of vitamins, trace and other elements.

Observation index before surgery $1 \mathrm{~d}$, the ninth day after morning weighed before blood sampling to detect albumin and lymphocyte count (LCC), the detection of immunoglobulin (Ig G, Ig $\mathrm{M}$, Ig A) and $\mathrm{T}$ lymphocyte subsets (CD4, CD8, CD4/CD8), observe the changes of these parameters before and after surgery. During nutritional support, daily monitoring of vital signs, recording without abdominal pain, bloating, diarrhea and vomiting and other gastrointestinal adverse reactions, before and after surgery to detect liver and renal function blood biochemistry, and observe the anus recover exhaust, defecation. The number of days of hospitalization calculate the number of cases of postoperative complications, the average cost of hospitalization and medical expenses.

\section{Brings A Full Range of Health Education To Patients}

Health education can not only improve the quality of life of patients, it is possible to greatly expand the connotation of intubation. Strengthen the patients or their families' right to know "," right to choose "to promote" less expensive, less painful, the effect is good, "the diagnosis and treatment, to promote empathy for the interests of the patients, efforts to reduce health care costs. They should patiently health education intubation, especially in patients who for the first time to the hospital, you should carefully explain matters intubation attention to them, the situation may occur during treatment with the medical staff how operating introduce cannula method, treatment, side effects and precautions in order to greatly relieve the patient of fear, stress and other psychological pressure. In addition, since the patient's family conditions, living environment, cultural level, status, occupation, age are different, natural health education for the ability to accept there is a big difference. In response to this phenomenon, we can not adopt a unified health education, should do individualized instruction.

Postoperative side of the nasal gastric tube inserted into the nose and intestine, oxygen tube insertion side will feel difficulty in breathing, throat pain and discomfort, prone to conflict, psychological anxiety. Former nurse preoperative enteral nutrition to patients to explain in plain language the meaning, importance, indicating retention nose intestine is an important guarantee for the implementation of enteral nutrition; Early enteral nutrition after surgery, the patient because there is no food by mouth, there is hunger, nurses should reassure patients and inform adaptation of early enteral nutrition needs $1 \sim 2 \mathrm{~d}$, and encourage patients to eliminate concerns and actively cooperate with treatment.

Intestinal and nasal gastric tube inserted from the side of the nose, no matter which of the catheter slide, will affect the treatment and care, it is particularly important conduit fixed, $3 \mathrm{M}$ tape can be cut into "workers" shape, on top of a stick in the nose on both sides, following a nose wrapped intestine and stomach, such fixation both beautiful and strong; duct tape affixed to the outer end of the outcrop, superscript insertion depth, each class to observe and record the length of the nose twisted intestine in vitro and the availability of parts, and prolapse Are coiled pipe in the mouth, if slippage timely reporting of medical treatment; $20 \mathrm{ml}$ rinse with warm water pipe after each infusion of nutrient solution injection port shall medication pills to grind into powder, fully dissolved after injection, and then warm flush water pipes; after infusion tube plugs seal the nozzle, properly fixed. The group of catheter slippage occurred.

Properly fixed: prevent detached, discounts, insurance holding feeding tube patency is an important guarantee for the implementation of enteral nutrition. Nose butterfly elastic tape fixed for various operations, stand up properly when placed nose intestine, placed stretch, extrusion, accurate placement depth nose intestine, so after the shift adjustment. 
Prevent clogging: Keep feeding tube patency, before each infusion of nutrient solution, both with warm water drip after 20 60 ml red tube, in order to avoid condensation of liquid nutrition plugging. In case of plugging, pressure injection with a syringe of saline or warm water, if pressure washing or not smooth, may be the first wife of a steel spiral guide wire nasal feeding tube proximal intestinal smooth entrance into it. When the guide wire placement, not fully entered, the tail should be left $2 \mathrm{~cm}$, so as not to prick from the feeding tube catheter side holes, damage the intestinal mucosa. Then saline, most patients can thus feeding tube blockage recanalization. As these two methods can not make feeding tube recanalization, indicating feeding tube distortion, discounts or spiral, only this time to extubation, stopping enteral feeding tube, the parenteral route nutritional supplements.

Prevent ectopic infusion: Do not enter wrong enteral nutrition blood vessels, enteral nutrition and intravenous infusion line tubing should hang eye-catching signs to show the difference, and two IV pole hanging separately.

Both sides of the nose catheter patients were forced to use mouth breathing, and the nutrient solution without oral intake, reduce the secretion of saliva, oral mucosa and tongue so dry and prone to oral infections. Therefore, to strengthen oral care, every day wash out your mouth with saline cotton ball or rinse with water 3 to 5 times, to keep your mouth moist. The group of oral infections occurred.

When the early postoperative period (24 h later), although the colon is still in a state of paralysis, but intestinal motility has been restored, as long as the patient hemodynamically stable, and with appropriate enteral feeding route, early enteral nutrition is fully implemented feasible [4], and promote the recovery of bowel movements, so within $24 \sim 48 \mathrm{~h}$ after surgery began enteral nutrition. Allow the patient's condition when receiving enteral nutrition for the semi-supine position, and 30 min after nasal remain in this position, to prevent nutrient solution reflux, cough and nasal gastric tube caused bowel prolapse; perfusion rate from slow to follow fast, perfusion from less to more, the concentration of the nutrient solution from low to high principle, the first infusion of 5\% sugar, salt, drip $15 \mathrm{gtt} / \mathrm{min}$, such as patients with no nausea, bloating, abdominal pain, diarrhea, nutrients may be given the following day was $20 \sim 40 \mathrm{ml} / \mathrm{h}$, not more than $120 \mathrm{ml} / \mathrm{h}$, the daily $500 \sim 1500 \mathrm{ml}$; the routine use of infusion warmer line feeding tube local heating to $37 \sim 40 \mathrm{e}$, too cold, too hot to stimulate the intestinal tract are caused by abdominal pain diarrhea; infusion of nutrient solution suspended in the IV pole and hung striking red card feeding, parenteral nutrition strictly separated areas, to avoid confusion; open nutrient solution using no more than $24 \mathrm{~h}$, infusion strictly Note aseptic liquid nutrition to avoid contamination; flatus tube removed, the small amount of liquid, such as patients with abdominal pain, bloating and discomfort, the whole amount of liquid into the next day, 2 3 d backward semi-liquid, without condition changes in another $2 \sim 3 \mathrm{~d}$ can be removed nose intestine, completely oral feeding. The group was 9 14 d plucking nose intestine.

Observation of diarrhea and care enteral nutrition can lead to abdominal surgical ward, including up to $30 \%$, up to $60 \%$ in the intensive ward. The reason is more complex, such as nutrient solution contaminated infusion too fast, bowel hyperactivity, nutrient solution temperature abuse, poor self-tolerance. To this end, the start of enteral nutrition infusion rate to avoid excessive; join Zhengchangsheng powder inside the capsule can be nutrient solution, dissolve completely shake infusion Zhengchangsheng can maintain the intestinal homeostasis; diarrhea, stool frequency recording and stool, and immediately send stool samples for testing, according to test results symptomatic treatment, and make the perianal skin care, stop treatment without effect of enteral nutrition. 5 cases of enteral nutrition on the first day of diarrhea, stool frequency 5 times/d, brown or yellow, watery, to slow down the infusion rate, the appropriate use of antispasmodic drugs, diarrhea gradually improved.

Observation of bloating and gastrointestinal motility patient care and poor nutrition infusion too fast, excessive use can cause bloating. Be slowing the infusion rate, appropriate to reduce the amount of nutrient solution instead of intermittent infusion or under doctor's orders, some patients can relieve itself; such as constipation, bloating caused by the administration enema anus, keep stool; gastrointestinal motility poor were given gastrointestinal drugs (such as domperidone). 
Early stomach after gastrectomy nursing role in the past that, in order to implement enteral nutrition after abdominal surgery should be back to normal gastrointestinal function. Studies have shown: the small intestine movement, digestion and absorption function even after a few hours to restore the colon in open surgery, 6 8 h intestines can accept input material, large intestine function 3-5 d recoverable, which makes early enteral nutrition possible. Our data show that, under the premise of careful observation and care, total gastrectomy between early postoperative jejunal enteral nutrition is safe and feasible. In this study, 93.3\% of the patients can tolerate input early enteral nutrition, only 1 patient had mild nausea and bloating, by adjusting the input speed after symptoms. EN group had no vomiting, diarrhea, abdominal pain and other symptoms, has not found serious complications such as anastomotic leakage, postoperative emptying patients in EN group was significantly less than PN group showed total gastrectomy, jejunal interposition between postoperative patients can be resistant by early enteral nutrition and enteral nutrition can promote the recovery of gastrointestinal function role. EN group were significantly higher than the serum prealbumin PN group, indicating early enteral nutrition can improve the body's protein metabolism, suppress decomposition, can promote the synthesis of an early improvement in the nutritional status, reducing the incidence of postoperative complications in this study group EN The complication rate was significantly lower than the PN group. Early enteral nutrition with a nutrient-rich, low cost, ease of use, physiological processes, to ensure a stable internal environment, inter total gastrectomy jejunal early postoperative nutritional support preferred method. Strengthening care is to ensure the nose intestinal smooth implementation of enteral nutrition therapy, the key to reducing complications. As long as the strict implementation of aseptic operation, close observation of clinical manifestations vary, vary over time, strict control of enteral nutrition infusion temperature, velocity and concentration. Strengthen the mission of enteral nutrition knowledge, and serious complications are completely avoidable.

\section{Conclusion}

Total gastrectomy surgery in patients with low immune function, can increase the risk of infection. Early enteral nutrition helps maintain intestinal mucosal barrier function, improve the patient's immune system, reduce infection rates, in favor of anastomotic edema, fistula prevention and treatment to reduce complications and improve the cure rate; enteral nutrition meet the body's physiological needs, at an affordable price, easy to operate, less side effects, patients and their families tend to accept. Care focuses attention to psychological care, good nose bowel care, strictly controlled input of nutrient solution, speed, temperature, nutrition liquid infusion process complications observation and care.

\section{References}

[1] Zheng Hong, Jiang Zhiwei. nutritional support in patients with gastrointestinal cancer [J].Parenteral and Enteral Nutrition, 2002,9 (4): 238-240.

[2] Slaviero KA, Read JA, Clarke SJ, et al.Baseline nutritionalassessment in advanced cancer patients receiving palliativechemotherapy [J]. Nutr Cancer, 2003,46 (2): 148-157.

[3] Hu Shuangjiu, Ma Xiaoqing. gastric cancer efficacy of early postoperative enteral nutrition and parenteral nutrition comparison [J]. Chinese Medicine Innovation, 2011,8 (9): 36.

[4] Lin Feng, Wang Zhidu. Postoperative enteral nutritional support in patients with gastrointestinal cancer [J]. Chinese Journal of Practical Surgery, 2007,22 (11); 661.

[5] Wang Chunrong, Chen Si, Jin Tao. Reasonable to reduce postoperative gastric cancer patients using enteral nutrition complications in the role of nutrition infusion pump [J]. Chinese Journal of Clinical Nutrition, 2007,388,15 (6): 388. 